\title{
Hacia la enseñanza de lenguas basada en tareas
}

\section{Christopher N. Candlin}
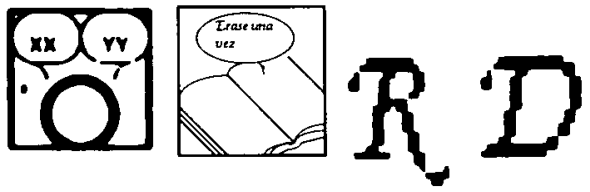

Se exponen en este artículo los principios que deben regir la programación de tareas en la enseñanza de lenguas extranjeras y se ofrecen vias para su implementación.

\section{INTRODUCCION}

Este documento toma como punto de partida la distinción que Candlin (1984a) propone entre las directrices del currículum y el diseño de programas. La distinción que allí se hace entre una programación estratégica y una táctica justifica la introducción de la tarea como unidad básica de la actuación en el aula. Sin embargo, las tareas no son simplemente unidades para la organización de lo que hemos denominado programas procesuales o de procedimientos (Prabhu, 1983; Breen, 1984). Son unos medios consistentes y apropiados para entender ciertos principios característicos de la enseñanza y aprendizaje comunicativo del lenguaje y sirven, además, como campo exploratorio de hipótesis para la pragmática y la adquisición de segundas lenguas. Pero las tareas deben definirse lo mismo que deben justificarse las maneras de operacionalizarlas, de modo que habrá que ofrecer ideas para su organización y para la clarificación de sus objetivos. Y, sobre todo, no se les puede otorgar un papel central en el programa sin antes evaluar cómo pueden ser seleccionadas y secuenciadas según unos principios determinados. Lo que vamos a exponer no podrá considerarse como una conclusión definitiva al respecto, pero habrá sido útil si ha servido para recalcar que el aprendizaje de lenguas basado en un enfoque por tareas no sólo favorece la adquisición y la comunicación en el aula, sino que también constituye un medio para el desarrollo de los programas de aula. Los temas que vayan surgiendo son, por sí mismos, cuestiones que el aprendizaje por tareas está especialmente dotado para tratar. 


\section{LAS DIRECTRICES DEL CURRICULUM Y LOS DISEÑOS DE PROGRAMAS}

En Candlin (1984) propongo la planificación del aprendizaje y la ensenanza del lenguaje a dos niveles (de los cuales ninguno se halla a nivel de programa según la definición tradicional) y donde la planificación presenta dos interpretaciones distintas. A nivel de las directrices del currículum (véase Figura 1) encontraríamos afirmaciones sobre el aprendizaje general y sobre el aprendizaje de un tema en particular, indicaciones sobre el propósito y la experiencia de aprendizaje, objetivos y formas de evaluación, relaciones entre el papel del profesor y el de los aprendices, así como bancos de items y guiones del aprendizaje acompañados de una relación de los procedimientos para diseñarlos, examinando y ampliando sus límites. En este proceso, llevado a cabo en el aula, los programas emergen a modo de planificaciones realizadas conjuntamente entre profesores y aprendices, registrando el qué, el cómo y el porqué. Ese constituye pues el nivel de la explotación táctica del principio estratégico. Tal planificación trata asuntos como qué se va a hacer, qué cuestiones se proponen, qué procesos se consideran más adecuados para examinar los problemas que surjan, qué información adicional se necesita y qué acciones en particular merece la pena llevar a cabo. Este nivel de toma de decisiones cotidiana, realizado en cooperación, desemboca en tres tipos de programación, no solamente en uno: Un programa de aprendizaje (y especialmente el aprendizaje de lenguas), un programa de contenido y un programa de acciones, de lo que se ha explorado y de cómo se ha alcanzado. En consecuencia, lo que tenemos es, como indica la Figura 1, un proceso dialéctico entre el nivel de directrices y el nivel de los programas, a través de los cuales las programaciones del aula pueden provocar cambios en el currículum.

Prospectiva

Principio estratégico

Planificación Nivel 1

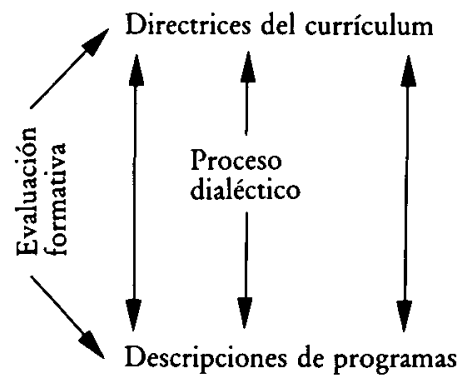

de aprendizaje

de conténido

de acciones

Acción Táctica

Planificación Nivel 2

Retrospección

$+$

Prospección 
Estas descripciones etnográficas y etno-metodológicas no evalúan únicamente la actuación en el aula sino que también proporcionan datos para estimar la viabilidad de las directrices mismas del currículum; son formativas a ambos niveles. De esas descripciones cabe esperar obtener información sobre los objetivos de aprendizaje acordados, el contenido elegido para trabajar y la forma de llevar a cabo dicho trabajo junto con la información y los recursos que se necesitaron y diseñaron, cómo se evaluó el trabajo y qué tipo de documentación fue seleccionada. En suma, una relación descriptiva de lo que ocurrió, sujeta sólo a las restricciones del comentario de Stenhouse: «there is no telling it as it is» (no hay forma de contarlo tal como es) (Stenhouse, 1975). Es precisamente en este nivel táctico de planificación del programa donde el concepto de tarea cobra significado, sugiriendo los medios para poder hacer una descripción de los distintos programas, como si se hiciera retrospectivamente. Las planificaciones del currículum y del programa, además de diferir a nivel de especificación y autoría, difieren también en su direccionalidad y en su descriptibilidad.

\section{TIPOS DE PROGRAMAS Y EXIGENCIAS DE LA TAREA}

Cada uno de los tipos de programa anteriormente descritos plantea sus propias exigencias sobre el diseño y el funcionamiento de las tareas de aprendizaje de lenguas. Al examinar dichas exigencias pueden también reiterarse algunos de los principios esenciales de un currículum comunicativo en la enseñanza, que naturalmente han sido ya ampliamente discutidos en otros escritos (cfr. Breen y Candlin, 1980; Breen, 1983; Johnson y Porter, 1983, entre otros).

\section{Exigencias del Aprendizaje}

Solamente cuando intentamos describir lo que ocurre con los aprendices y su proceso de aprendizaje en el aula de lengua es cuando la fácil suposición de que «los aprendices difieren entre sí» empieza a imponer unas exigencias considerables sobre el diseño de tareas. Sin duda los aprendices difieren entre sí en muchos aspectos, entre los cuales podemos citar: su inteligencia y su habilidad para aprender una lengua; sus intereses, necesidades, deseos y lagunas; sus estrategias para comunicarse y para interpretar lo que los interlocutores dicen o escriben; sus estilos, formas y distintos ritmos de aprendizaje; su deseo de participar en la dirección de su propio aprendizaje; su necesidad de corrección y supervisión; sus antecedentes socioculturales y educativos; sus diversas actitudes hacia la lengua meta y los motivos para aprenderla. A estas diferencias deberiamos añadir nuestra experiencia como enseñantes ( $y$ aprendices) respecto a aquellas condiciones que intensifican el aprendizaje en lugar de inhibirlo, sabiendo que cada una impondrá también sus propias condiciones en el momento de diseñar las tareas. Por ejemplo, es posible que tengamos el convencimiento de que los aprendices consiguen mejores resultados cuando son conscientes de lo que tienen que lograr y de cómo van a juzgarse sus esfuerzos, cuando ellos mismos han tomado parte en la especificación de ambas cosas. Es posible que creamos que no podemos esperar que cada aprendiz aprenda lo mismo, de 
la misma forma y al mismo ritmo, o bien que pensemos que las preferencias del aprendiz pueden influir tanto en la elección como en la tarea de aprendizaje, en los procedimientos y en los criterios de evaluación.

\section{Exigencias del contenido}

Al considerar el «contenido» de un currículum de enseñanza de lenguaje como un componente característico y básico para el desarrollo de la competencia comunicativa, debemos ahora (siguiendo los argumentos de Widdowson, 1983; Canale y Swain, 1980; Canale, 1983; Candlin, 1985, entre otros) incluir los dos términos conocimiento y procedimientos, no conocimiento solamente, re-evaluando después lo que se entiende por conocimiento. Como verenos, la definición a la que lleguemos tendrá sus propios efectos sobre el diseño de la tarea y su funcionamiento. En resumen, nuestra competencia comunicativa es en parte pragmática y en parte cognitiva. Incluye nuestra capacidad de crear significados, a través del «potencial de significados» de Halliday, mediante el cual hacemos uso de nuestra capacidad de negociar, interpretar y expresar valores, en lo que constituye un proceso educativo y creativo. Contempla lo que se aprende tanto desde el punto de vista de estructuras de conocimiento organizadas, como de los procedimientos necesarios para adaptar ese conocimiento a la solución de nuevos problemas de construcción de significados, aspectos que, hasta ahora no se habían contemplado conjuntamente. Tal conocimiento, como Widdowson (1983) indica, no consiste ya tanto en una lista de elementos, sino que se entiende como un conjunto de estructuras que incluyen formas, pero también organizaciones textuales y rutinas sociales profundamente conectadas a nuestros sistemas de valores y creencias y propias de unas situaciones culturales y sociales concretas. Los procedimientos son aquello que establecemos para trabajar sobre las estructuras de conocimiento, poniendo a prueba sus límites en el proceso de comunicación y haciendo uso de nuestra capacidad de inferir y percibir problemas, dando lugar a estrategias de resolución de problemas. Esa interacción entre conocimiento y procedimiento es de por sí una tarea que, como el aprendizaje, es sensible a la situación, y es intrínsecamente variable y singular.

\section{Exigencias de actuación}

Los dos tipos de exigencias indicadas anteriormente proporcionan un ambiente óptimo para el aprendizaje comunicativo del lenguaje: el aula, donde se alienta a los aprendices a responder activamente, explorar y participar en una comunicación útil con los compañeros. Tal como Breen $(1983,1985 a)$ indica, dicho aprendizaje constituye una actividad favorablemente compartida donde los aprendices se hallan dentro de un proceso negociador, consigo mismos en relación a lo que ya saben, con los demás, pues deben compartir y perfeccionar su conocimiento, y con el contenido del currículum, en términos de lo que debe aprenderse. En un aula de tales características impera una condición muy clara para la actuación: la necesidad de ofrecer a los aprendices una variedad de opciones alternativas y diferenciadas en lo que respecta a la elección del tipo de actividad en el aula, materias y formas de trabajar. Es por ello indispensable ser coherentes: si creemos que el aprendizaje de una lengua es más efectivo cuando los 
aprendices se hallan dentro de una actividad de exploración y experimentación conjunta, aportando sus contribuciones personales, entonces no podemos negarles, en el momento de organizar esas actividades, la variedad que tales contribuciones comportan. Sin embargo, las exigencias del aprendizaje y los programas de contenido imponen unas restricciones más específicas en la acción que se realiza en el aula. ¿Qué limitaciones imponen los recursos de gestión en el aula sobre los posibles cambios de datos variables, distintas peticiones de información y actividad diversa? ¿De qué forma pueden diseñarse las tareas a fin de que proporcionen un máximo de oportunidades de elección tanto para el aprendiz como para el profesor? ¿Cumplirán esas tareas con los requisitos de concreción que las instituciones requieren y que son causa de cambios en el currículum?

\section{CONDICIONES DEL APRENDIZAJE DE LENGUAS BASADO EN TAREAS}

A partir de lo que se ha dicho hasta el momento aflora un conjunto de características relativas a la enseñanza comunicativa del lenguaje que impone unas condiciones sobre el diseño de tareas. Ofrecer ahora un resumen puede ser de utilidad.

Las tareas crean condiciones para los siguientes aspectos:

a) Exploración del lenguaje y del aprendizaje por parte del aprendiz.

b) Desafío y crítica de la lengua, del aprendizaje y del programa por parte del aprendiz.

c) Negociación del lenguaje, del aprendizaje y del programa por parte del aprendiz.

d) Interacción e interdependencia entre aprendices y enseñantes, y entre datos, recursos y actividades de aprendizaje de una lengua.

e) Creación de listados tácticos como medio para evaluar el lenguaje, el aprendizaje y la acción, y como medio para criticar las directrices del currículum.

f) Provisión del input comprensible y procedimientos para utilizarlo.

g) Acomodación de las diferencias entre los aprendices.

h) Posibilidad de formular problemas sobre la lengua, el aprendizaje y la acción en el aula.

i) Gestión del aprendizaje de una lengua.

A partir de estas condiciones podemos extraer algunos criterios para lo que podrían denominarse «buenas» tareas de aprendizaje de lenguas. A continuación se presentan dos listas basadas en tales criterios. La primera procede de un curso de formación permanente para formadores de profesores de lengua en Europa; la segunda de una clase de licenciados de la Universidad de Hawai, también profesores de lenguas. Ambas listas se centran en el aprendizaje de una lengua y utilizan una actividad presentada por primera vez por Michael Breen y el autor de la Convención de TESOL 1982, en Honolulú, Hawaii. 
Criterios para las «buenas» tareas de aprendizaje de lenguas

1. Lista (Nijmegen, Holanda, septiembre de 1985)

- Equilibradas: entre un resultado abierto y los recursos limitados del aprendiz.

- Motivantes: que requieran participación y sean interesantes, desafiantes, gratificantes y valiosas.

- Co-operativas: que desarrollen destrezas sociales y destrezas de gestión en el aprendizaje.

- Estratégicas: que estimulen el desarrollo de estrategias personales para el aprendizaje de lenguas.

- Diversas: variadas según los distintos niveles, destrezas, estilos y objetivos de los aprendices.

- Convergentes: sin ambigüedad, con objetivos claros y relevantes a las necesidades y metas de los aprendices.

- Abiertas: extensibles, permeables, accesibles por y para los aprendices.

- Estructuradas: graduables, variables, ordenables, que ofrezcan la posibilidad de ser organizadas según tiempo, lugar y disponibilidad de los aprendices.

- Críticas: evaluativas, formativas, que ofrezcan feedback y planteen problemas.

2. Lista (Honolulú, Hawaii, Primavera de 1984)

1. Deberían fomentar la atención al significado, a los propósitos y a la negociación.

2. Deberian estimular la atención a datos relevantes.

3. Deberian proponer objetivos a partir de las necesidades de los aprendices.

4. Deberian tener en cuenta enfoques flexibles a la tarea, ofreciendo rutas, vehículos, modos de participación y procedimientos distintos.

5. Que puedan tener en cuenta soluciones diversas según las destrezas y estrategias de los aprendices.

6. Deberian incluir las contribuciones, actitudes y sentimientos de los aprendices.

7. Deberían ser desafiantes, aunque no amenazadoras, para fomentar la toma de riesgos.

8. Deberían requerir un input de todos los aprendices con relación al conocimiento, destrezas y participación.

9. Deberian poder definir un problema en el que todos los aprendices trabajaran, y deberían estar centradas en los aprendices aunque guiados por el profesor.

10. Deberían requerir el uso de la lengua para la resolución de la tarea.

11. Deberían tener en cuenta la co-evaluación del aprendiz y del profesor, de la tarea y de la ejecución de ésta.

12. Deberían desarrollar capacidades en el aprendiz para que éste pueda valorar las consecuencias y repercusiones de la tarea en cuestión.

13. Deberian proporcionar oportunidades de metacomunicación y metacognición. 
14. Deberían proporcionar oportunidades para la práctica del lenguaje.

15. Deberían facilitar la formación del aprendiz en percibir y resolver problemas.

16. Deberían fomentar el intercambio de información y de destrezas.

17. Deberían proporcionar la corrección y el feedback necesarios, para los aprendices y para las tareas.

18. Deberían elevar el grado de toma de conciencia de los aprendices y la capacidad de reflexión.

19. Deberían fomentar una conciencia crítica sobre los datos y los procesos del aprendizaje de la lengua.

20. Deberían ofrecer una elevada gratificación al esfuerzo invertido y que éste, a la vez, mereciera la pena.

Es evidente que ambas listas de criterios se pueden superponer, reestructurar y ampliar. Se les podría atribuir tres méritos: en primer lugar, proceden de un control experimental del comportamiento en las tareas; en segundo lugar, tratan muchos de los aspectos relacionados más arriba bajo las exigencias de aprendizaje, contenido y actuación; y en tercer lugar, sugieren formas de determinar los componentes de las tareas y de implementar las tareas del currículum de la enseñanza del lenguaje. A otro nivel, puede observarse que tratan algunos aspectos básicos de otras disciplinas que también intervienen en el aprendizaje y enseñanza de lenguas: de la pragmática, por el énfasis que se da a la negociación de valores y a la metacomunicación crítica; de la adquisición de una segunda lengua, por el énfasis dado al input y a la interacción, a las estrategias de aprendizaje y a la concienciación reflexiva; de la gestión en el aula, por la importancia que se concede a los datos, la información y la actividad, a la participación y diversificación flexible, a la evaluación y a la eficacia. Además, el significado que se otorga al proceso de comunicación entre los participantes, a través de una diversidad de formas y medios dirigidos a la actuación y al control de esa acción es coherente con los principios característicos de una enseñanza comunicativa del lenguaje, tal como señalo al comienzo de este documento.

\section{UNA DEFINICION DE TAREA DENTRO DEL CONTEXTO DE APRENDIZAJE DE LENGUAS}

A partir de las condiciones anteriormente mencionadas y los criterios que se han detallado, podríamos llegar a la siguiente definición básica de una tarea de enseñanza-aprendizaje:

Una actividad perteneciente a un conjunto de actividades distinguibles entre sí, secuenciables, capaces de plantear problemas y que involucran a aprendices y profesores en una labor conjunta de selección, a partir de una gran variedad de procedimientos cognitivos y comunicativos aplicados a un conocimiento, existente o nuevo, y de la exploración y búsqueda colectiva de fines predeterminados o emergentes dentro de un medio social.

No es de extrañar que haya reacciones por parte del lector y también será distinta la importancia que se dé a cualquier definición de características similares; y así es como debiera ser, ya que cada aula y cada programa configurarán unos rasgos particulares dentro de su esfera práctica, ya sea 
en general o en aspectos concretos. Por ello una actividad útil en un curso de formación permanente de profesorado examinará precisamente esa valoración tan amplia: la referente a la definición anterior y la que tenga que ver con las condiciones de un aprendizaje de lenguas basado en un enfoque por tareas, de las que se ha hecho una relación más arriba. Y a pesar de que se deje de lado el tipo de énfasis u orientación de las tareas, en cada caso convendría especificar las siguientes características clave:

- Input.

- Papeles.

- Escenarios.

- Acciones.

- Corrección.

- Resultados.

- Feedback.

Podemos glosarlas de la siguiente forma:

\section{a) Input}

Con ello entiendo cualquier tipo de datos a trabajar que presenten los aprendices o enseñantes, en cualquier medio o medios y relacionados con cualquier experiencia personal de los participantes. ¿Qué recursos son necesarios para llevar la tarea a buen fin?

b) Papeles

Con ello entiendo la especificación de los papeles de los participantes en relación al cumplimiento de la tarea y sus papeles en lo que concierne a sus relaciones entre sí. ¿Qué es lo que tienen que hacer los participantes y quién asigna tales deberes? ¿Cómo se espera que los participantes cooperen? ¿Cuál debe ser su distancia relativa y su potencia relativa? ¿Quién participa y quién controla, quién instruye y quién guía?

c) Escenarios

Con ello me refiero a la disposición del aula según requiera la tarea. ¿Implica un trabajo individual, por parejas o en grupo? ¿Qué combinaciones o secuenciación? ¿La elección del escenario de trabajo se deja a libre albedrío de los participantes o se estipula previamente? ¿Qué vínculos hay entre el conjunto del escenario del aula y la actividad fuera de ella?

\section{d) Acciones}

Con ello entiendo los procedimientos a seguir para la comprensión, ejecución y finalización de la tarea. ¿Cómo se establecen? ¿La elección de los procedimientos se deja abierta o se determina con anterioridad? ¿Cómo va a compartirse el trabajo? ¿Qué comportamientos se esperan y se van a tolerar de los diversos participantes? ¿Quién hace qué con qué con quién y cómo?

\section{e) Corrección}

¿Cómo se va a corregir y registrar la selección del input, la elección de papeles, la adopción del escenario de trabajo y la eficacia de la acción? 
¿Quién se hace cargo de este papel? ¿Cómo debe realizarse la corrección? ¿Cómo van a captarse los cambios en la dirección de las alternativas del input, del escenario, de los papeles o de la acción? ¿Dónde debe centrarse la corrección en la comunicación, en el aprendizaje, en el comportamiento social?

\section{f) Resultados}

Los fines de la tarea. ¿Deben establecerse previamente o pueden irse perfilando o reformulando? ¿Es posible, está permitido llegar a resultados diversos? ¿Pueden los participantes definir los criterios para el cumplimiento de la tarea durante el mismo proceso? ¿Qué nexos deben o pueden establecerse con otras tareas? ¿En qué términos deben exponerse los resultados?

\section{g) Feedback}

Entiendo con ello la evaluación de la tarea. ¿Quién la proporciona a quién y en qué momento? ¿Está ese feedback implícito en la tarea misma, permitiendo por ejemplo la realización de tareas secundarias, o es independiente? ¿Contempla a la vez el contenido y el proceso de la tarea? Siendo así, ¿cómo se formulará el feedback de ese último? ¿Cómo se conectan las tareas con las experiencias sociales y cognitivas de los aprendices? ¿Cómo puede adaptarse el feedback a los cambios en el comportamiento del aprendiz de una forma adecuada?

Este desglose es también limitado y parcial. Los términos pueden volverse a formular rápidamente, de acuerdo a un público y móviles distintos. En el Manual del Profesor del paquete multimedia Challenges (Candlin y Edelhoff, 1982), simplificamos las características de los criterios como si se tratase de un conjunto de retos para el diseño del programa. Puede verse a continuación:

- ¿Pueden los aprendices elegir entre diversas tareas alternativas para fines comunicativos que ellos mismos ayuden a especificar?

- ¿Pueden los aprendices elegir entre diversas rutas alternativas dentro de una tarea, escogiendo entre distintos medios y modos de trabajo?

- ¿Pueden los aprendices utilizar las diversas contribuciones propias para realizar cualquier tarea?

- ¿Pueden elegir los aprendices contenidos diversos para trabajar en una tarea?

- ¿Pueden sugerir los aprendices sus propias formas de evaluar una tarea y su actuación en ella?

- ¿Pueden los aprendices diseñar sus propias tareas?

A pesar de estar motivadas por los principios del currículum general, las características que acabamos de mencionar requieren el soporte de aquellos programas tácticos que mencionábamos al principio de este documento. Los informes recientes de Dam (1985) nos ofrecen más medios para determinar las características de las tareas del aprendizaje de lenguas dentro de unos esquemas comunicativos. Dice así:

El objetivo central de mi enseñanza podría ser definido como «autonomía», basada en construir a partir de la propia planificación del proceso de 
enseñanza/aprendizaje de los alumnos y del desarrollo/extensión de su toma de conciencia sobre los objetivos y responsabilidad en el proceso.

En este enfoque, el alumno, solo, o los alumnos juntos, siempre que desean utilizar al profesor como consejero/asesor:

- establecen de forma independiente los fines de un determinado período de enseñanza/aprendizaje;

- eligen independientemente los materiales, procedimientos y sistemas de evaluación;

- se hacen responsables de juzgar si se ha logrado una meta y qué hacer a continuación, de forma independiente.

La evaluación se contempla como el eje del proceso de enseñanza/aprendizaje en donde las charlas que surjan entre el profesor y los alumnos, o entre los alumnos entre sí son:

- ¿qué estamos/estoy haciendo?;

- ¿por qué lo estamos/estoy haciendo?;

- ¿cómo lo estamos/estoy haciendo?;

- ¿para qué puede utilizarse?;

Terminaré dirigiendo la atención a dos características esenciales en este enfoque:

- los estudiantes/profesores deben aprender a aceptar el hecho (tener la seguridad de) que los alumnos quieren aprender;

- los estudiantes/profesores deben recibir la oportunidad de observar a "los alumnos en el trabajo" con el fin de aprender lo mucho que puede «aprenderse» a partir de tales observaciones.

(Dam, 1985: 1 y 19)

Las reflexiones que este informe hace sobre las características del diseno de tareas de aprendizaje de lenguas son claras $\mathrm{y}^{\bullet}$ corroborativas a la vez. Un apoyo similar puede hallarse en los mismos comentarios de los alumnos (cfr. Dam, 1983, 1984), y de la lista de Raths (1971), citada en Stenhouse (1975), sobre actividades que merecen la pena. Esta que mostramos a continuación no está relacionada con ningún tipo de disciplina, y sería muy formativo que los lectores se planteasen su relevancia dentro del aprendizaje de lenguas, después de haber visto todo lo anterior:

1. Siendo todo lo demás igual, una actividad merece la pena más que otra si permite a los niños hacer elecciones con conocimiento de causa para realizar la actividad y reflexionar sobre las consecuencias de sus elecciones.

2. Siendo todo lo demás igual, una actividad merece la pena más que otra si asigna papeles activos, en lugar de pasivos, a los estudiantes en las situaciones de aprendizaje.

3. Siendo todo lo demás igual, una actividad merece la pena más que otra si requiere que los alumnos se comprometan con posibles interrogantes sobre las ideas, aplicaciones de procesos intelectuales o problemas actuales, ya sean personales o sociales.

4. Siendo todo lo demás igual, una actividad merece la pena más que otra si pone a los niños en contacto con materiales auténticos (objetos reales, materiales y artefactos).

5. Siendo todo lo demás igual, una actividad merece la pena más que otra si el resultado de la misma pueden cumplirla con éxito niños que difieran en su nivel de destrezas.

6. Siendo todo lo demás igual, una actividad merece la pena más que 
otra si requiere de los alumnos que examinen una idea, una aplicación de un proceso intelectual o un problema actual que haya sido previamente analizado.

7. Siendo todo lo demás igual, una actividad merece la pena más que otra si requiere que los alumnos examinen temas o asuntos que los ciudadanos de nuestra sociedad normalmente no examinan, y a los que normalmente la mayoría de medios de comunicación del país no prestan atención.

8. Siendo todo lo demás igual, una actividad merece la pena más que otra si involucra a los estudiantes y a otros miembros facultativos en la "toma de riesgos", no riesgos de vida o muerte, pero sí riesgos de éxito o fracaso.

9. Siendo todo lo demás igual, una actividad merece la pena más que otra si requiere que los alumnos tengan que revisar escritos y tengan que ensayar y pulir sus esfuerzos iniciales.

10. Siendo todo lo demás igual, una actividad merece la pena más que otra si hace que los alumnos se involucren en la aplicación y manejo de reglas, normas y disciplinas razonables.

11. Siendo todo lo demás igual, una actividad merece la pena más que otra si proporciona a los estudiantes la oportunidad de compartir la planificación, la realización de un proyecto o los resultados de una actividad con los demás.

12. Siendo todo lo demás igual, una actividad merece la pena más que otra si es pertinente para los propósitos expresados por los alumnos.

(Raths, 1971: 716)

Tres comentarios vienen al caso: en primer lugar, que muchos de los criterios de Raths coinciden con la experiencia que en las tareas tienen los profesores de lenguaje y los aprendices (por lo menos en un caso); segundo, que a pesar de no especificar área disciplinar alguna, los criterios de Raths en diversos casos son importantes en relación al aprendizaje de lenguas [por ejemplo, nótese la vinculación con el estudio de OISE, The good language learner (Naiman et al., 1978) y con el articulo de Rubin (1981) que trata las consecuencias de la enseñanza del lenguaje a partir de este estudio]; en tercer lugar, la forma en que estos criterios ofrecen la base de un programa de investigación en el campo del comportamiento comunicativo (pragmático y cognitivo) de los aprendices de lenguas y su adquisición.

\section{UNA TIPOLOGIA DE LAS TAREAS DE APRENDIZAJE DE LENGUAS}

Aunque Raths, por ejemplo, ofrece unos medios que permiten diseñar tareas y distinguirlas entre sí, los criterios que ya hemos indicado, no proporcionan más que sugerencias implícitas sobre la posibilidad de catalogar las tareas en diversos tipos. Tal tipología será necesaria para cualquier currículum que tenga distintos fines. Será importante en la enseñanza del lenguaje y también en otras áreas donde los participantes puedan centrarse en diversos aspectos del currículum. Por ejemplo, es posible que nuestro centro de interés sea el proceso de resolución de problemas, o el desarrollo de destrezas de aprendizaje, la distribución de información, la experimentación o la producción discursiva, entre otros. En un aula que funcione bajo un enfoque por tareas, lo más probable es que sea necesario hacer alguna que otra selección, apropiada y con la información debida, del banco de ta- 


\section{4}

reas asequibles. Si se compara el aula con la red de educación del lenguaje integrado (integrated language education network) propuesto por Leech y Candlin (1986), se ampliaría un banco de datos (textos) por medio de un conjunto de recursos informativos para facilitar el acceso a esos textos, y se obtendrían los aspectos más relevantes a través de la agencia de un banco de tareas de proceso, utilizando un programa patrón para llevar a cabo las conexiones necesarias según lo requiera el aprendiz.

¿Qué posibilidades hay para una tipología de tareas? A continuación les propongo cuatro sugerencias que expongo brevemente. Habrá seguramente más tipos, y, dentro de ellos, un sinfín de muestras imaginativas ajustables a los distintos grupos y categorías de aprendices. Sin duda puede haber todavía otras áreas de desarrollo del currículum, que puedan examinarse con provecho por medio de las tácticas de programa a las que ya he hecho alusión. Pero hay dos advertencias: aunque podamos etiquetar algunas tareas concretas como pertenecientes a un tipo u otro, no puede asegurarse que una tarea de un tipo no pueda ofrecer interés, por su experiencia, dentro de la esfera de otro. Está claro que las tipologías no pueden tener límites claros, ni por exigencias de gestión, por muy necesario que eso sea. Por otra parte, cualquier tipología se irá perfilando a medida que la tarea avance y se vaya evaluando. Aunque las tareas, sean especialmente adecuadas para promover una u otra conducta (por tener un enfoque bien pragmático, bien cognitivo), irán tomando valor por resultados distintos a los concebidos en principio. En segundo lugar, la categorización de los tipos de tarea no es arbitraria. Las propuestas de organización de tareas de autores como Prabhu (1983), Long (1984), Waters y Hutchinson (1983) muestran una amplia variedad de aproximaciones a la clasificación de tareas: algunas valoran un objetivo de conducta (del mundo real), otras, de estrategias cognitivas, otras la ejecución comunicativa, otras procesos generalizados, otras en fin, las mismas estructuras sociales del aula. Hay sin duda un vasto campo por donde moverse antes de llegar a algún acuerdo.

Tipo 1. Enfasis en la formación del aprendiz (Cfr. Wenden, 1985).

a) Tareas de concienciación:

1. Tareas centradas en cómo funciona el lenguaje.

2. Tareas centradas en la manera en que los aprendices reaccionan a la enseñanza del lenguaje.

3. Tareas centradas en las creencias personales sobre el aprendizaje de lenguas.

b) Tareas de necesidades, objetivos y recursos:

1. Tareas centradas en lo que tiene que aprenderse.

2. Tareas centradas en cómo debe aprenderse.

3. Tareas centradas en los recursos necesarios para 1) y 2).

Tipo 2. Enfasis en el intercambio de información (Cfr. Candlin y Edelhoff, 1982).

1. Información e Puesta en común ideas propias de $\rightarrow$ de las ideas de cada aprendiz cada aprendiz

Generación de

$\rightarrow$ ideas variadas y soluciones 
Aquí las tareas se centran en las ideas y la información procedente de cada uno de los aprendices del grupo. La puesta en común de dichas ideas se realiza sin ningún proceso de selección o sintesis.
2. Información
e ideas
propias de
cada aprendiz
Puesta en
$\rightarrow$ común de las
$\rightarrow$ ideas de
cada aprendiz
$\rightarrow$ Compromiso
$\rightarrow$ Unica respuesta correcta

Aquí el énfasis recae sobre la importancia dada al resultado: ya sea una decisión acordada por el grupo o bien una única respuesta aceptable.

3. Contribución individual de la información dada

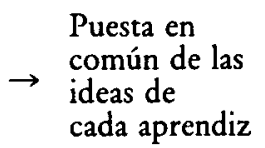

$\rightarrow$ Compromiso

$\rightarrow$ Unica respuesta correcta

Aquí se da un tipo de información distinta a cada aprendiz. La resolución del problema radica en el buen engranage de las distintas parcelas de información con el resto de los aprendices, del grupo o de la clase.
4. Hallazgo
individual
de distinta
información
Puesta en
común de las
$\rightarrow$ ideas de
cada aprendiz
$\rightarrow$ Compromiso
$\rightarrow$ Unica respuesta correcta

Aquí, cada aprendiz es responsable de hallar individualmente la información de un problema o una materia de trabajo y transmitir sus datos al resto del grupo. El producto final de la actividad recopila los diversos resultados e información procedentes de cada uno de los aprendices.

Tipo 3. Enfasis en la investigación y experimentación (Cfr. Greve, 1981).
a) Tareas diseñadas para la exposición de pre- guntas y problemas iniciales
b) Tareas diseñadas para la formulación de hipó- tesis
c) Tareas para determinar métodos y procedi- mientos
d) Tareas para determinar un plan de trabajo preciso....
e) Tareas diseñadas para evaluar los resultados...
¿Qué? ¿Por qué?
¿Cómo?
¿Cómo de bien? ¿Por qué? ¿Qué? ¿Cómo? ¿Para quién? ¿Dón- de?

Tipo 4. Enfasis en las estrategias del aprendiz (Cfr. Bruner, 1974).
a) Tareas centradas en el reconocimiento y per- cepción de problemas
b) Tareas centradas en la selección, clasificación y estructuración.
c) Tareas centradas en la inferencia, deducción y apreciación
d) Tareas centradas en la aplicación de los resul- tados de datos nuevos

Atención

Razonamiento

Ir más allá de lo dado

Transferencia y generalización 
Tal como indico más arriba, no sería difícil concebir otros tipos. Por ejemplo, la tipología de estrategias de comunicación que se halla en Faerch y Kasper (1983), proporciona una base para el diseño de tareas de orientación comunicativa, mientras que las propuestas de concienciación de procesos gramaticales que se da en Sharwood-Smith (1981) y Rutherford (1986), indican la forma en que las tareas pueden centrarse en aspectos concretos del sistema lingüístico.

\section{TAREAS Y FINES EDUCATIVOS}

Sea cual sea el tipo de tareas que se proyecten y las tipologías que se establezcan, la selección de cualquier recurso precisará un fundamento de mayor orden, además de los requisitos tácticos de las necesidades más inmediatas del aula. Ese fundamento lo han proporcionado en parte los criterios de diseño de tareas mencionados más arriba, y en especial a partir de la lista de Raths (1971). Sin embargo, dado el frecuente fracaso de aquellos currícula de lenguaje que enfatizan por encima de todo los fines educativos, en su empeño de conseguir un entrenamiento a bajo costo, merece la pena volver a señalar qué es lo que podría constituir ese conjunto de fines educativos. Sabido es que muy a menudo los objetivos de enseñanza de lenguas se establecen de forma externa a los aprendices, y al valor que tales objetivos tienen dentro del desarrollo educativo general del aprendiz. $\mathrm{Y}$, preocupados como estamos con el aprendizaje del lenguaje, es muy fácil olvidar que debiéramos ocuparnos con un grado similar, o mayor, por el desarrollo de la personalidad de nuestros aprendices. ¿Cómo pueden definirse esos fines educativos y convertirse en el centro de las tareas del aprendizaje de lenguas? Para empezar, podríamos distinguir los aspectos siguientes:

\section{Toma de conciencia}

Representa la oportunidad que ofrecen las tareas a los aprendices de volverse más conscientes de su propia personalidad y papel social, y de la de sus compañeros, en la medida en que participan en la comunicación. Las tareas pueden centrarnos en ver cómo el lenguaje se utiliza para reflejar y reforzar nuestros sistemas de valores y creencias, explorando críticamente cómo el lenguaje puede actuar tanto para clarificar como para oscurecer el proceso. Conciencia, también, de cómo actuamos nosotros y los demás para comunicarnos y aprender.

\section{Responsabilidad}

Si se sostiene, como hemos hecho aquí repetidas veces, la necesidad de ofrecer unas actividades alternativas y unos fines diferenciados, no podemos ignorar los problemas que surgen ante la necesidad de priorizar y seleccionar. Mantener la necesidad de interdependencia entre aprendices y enseñantes implica que ambos deben poseer una destreza para ejercitar la posible elección de actividades a emprender, afrontar los problemas y obtener el apoyo necesario. ¿De qué forma pueden diseñarse las tareas a fin de desarrollar tal destreza? Sería irónico que un programa basado en un enfoque 
por tareas solamente aportara a los alumnos experiencia en seguir unas pautas preestablecidas y no promoviera el desarrollo de su capacidad personal para diseñar sus propios proyectos.

\section{Tolerancia}

¿Qué nexos forjamos entre los mundos culturales y sociales de los aprendices externos al aula, y el mundo que hay dentro? La viabilidad y mejora de ambos mundos dependen de la mutua aceptación y tolerancia de sus miembros, y de la superación de las barreras interpuestas por las ideologías y los prejuicios. El reto de las tareas consiste aquí en adoptar una postura crítica, tanto en términos de elección de contenidos como de procesos. No debiera ser una elección regida únicamente por las consideraciones sociales comunes sobre algo a experimentar en un laboratorio, si con ello se ocultan las asimetrías y diferencias regulares de la interacción entre hablantes no-nativos y los nativos en el mundo real exterior. Eso seria tan válido para los procesos como lo es para el contenido.

\section{Autorrealización}

Sería difícil encontrar un conjunto cualquiera de directrices de currículum que no diera prioridad a la autorrealización y a la auto-afirmación del aprendiz. Sin embargo, podemos imaginar tareas que no se centren en esas prioridades donde no se procura que los aprendices hagan valoraciones o practiquen elecciones; ni se les estimule a beneficiarse de los errores o de las actividades inconclusas; ni donde tampoco encuentren oportunidad alguna de imprimir sus propias huellas en las tareas que se les presetan. Esos aspectos sí tienen gran importancia en tareas que persigan el desarrollo de la competencia comunicativa, donde la conciencia del aprendiz de lo que puede conseguirse por medio del lenguaje y la flexibilidad de éste para expresar actitudes e ideas constituye el núcleo del currículum.

\section{La confianza en uno mismo}

Cualquier conjunto de materiales basado en un enfoque por tareas corre el riesgo de desmoralizar o bien de aumentar la confianza de los aprendices en sí mismos. Además, a los diseñadores de tareas les es imposible calibrar por adelantado los umbrales de competencia de cada uno de ellos con exactitud. Lo que puede hacerse, como ya he sugerido antes, es ofrecerles rutas alternativas, cuya elección constituye la ocasión de negociar entre aprendices y enseñantes. Una vez embarcados en una selección de tareas relacionadas y dependientes entre sí, surgen oportunidades de. aumentar y poner a prueba la autoconfianza de los aprendices, a medida que van evaluando su propia actuación. Aquí, el fin de las tareas consiste en ayudar a que los aprendices se hagan conscientes de su creciente capacidad para comunicarse con los demás, y que, desarrollen la autoevaluación de su propio estado de aprendizaje. Sólo en el momento en que esta autoevaluación pueda conseguirse, en un ambiente de seguridad y sin presiones, es cuando puede promoverse un aprendizaje efectivo. A tal efecto, las tareas deben representar un desafío personal para los aprendices y asimismo ofrecerles apoyo por medio de recursos asequibles y etapas periódicas de verificación y corrección. 


\section{SECUENCIACION DE TAREAS}

Hablar de los programas basándose y criticando las directrices del currículum, como hemos hecho, implica por los menos que parte de la programación debe realizarse a nivel de tácticas en el aula, aunque sea a corto plazo, como necesariamente debe ser. Debemos además acometer la secuenciación que se ajuste mejor a las tareas. Si éstas sólo constituyeran meros vehículos para poder presentar unidades de sintaxis «empaquetadas" no importa lo hermoso del diseño, habría que discrepar contra cualquier tipo de secuenciación predeterminada en base a los resultados de investigaciones sobre la adquisición de una segunda lengua. Porque, a pesar de haberse demostrado empíricamente (para alguna serie de elementos restringidos) la existencia de un orden de adquisición natural, no hay pruebas que confirmen que todos los aprendices se hallan necesariamente en el mismo punto de ese orden en un determinado momento de la enseñanza. La consecuente variabilidad imposibilitaría cualquier solución, por sencilla que fuese. De la misma manera, si se tuvieran que diseñar las tareas según un análisis componencial de alguna conducta comunicativa determinada (como es el caso en la mayoría de diseños curriculares del E.S.P. "), no hay nada que nos permita asegurar que el orden de asociación de los componentes sea coherente para las necesidades de los aprendices. Si, por otra parte, estuviéramos dispuestos a abandonar en el empeño de toda pretensión de secuenciación preestablecida, para basarnos en el feedback, se requeriría un sistema de autoevaluación de los alumnos muy elaborado. Además, sería necesario que las directrices de un profesor en un mundo tan dinámico se basaran en unos principios que no fueran nada arbitrarios ni gratuitos.

¿Qué tipos de directrices basadas en principios podrían resultar? A continuación voy a mostrar unas cuantas que sin duda alguna son, una vez más, interdependientes y exclusivas, que pueden ser, en mayor o menor grado, clarificadas con ejemplos. Las directrices debieran estimular tanto la discusión como la experimentación, tan apreciadas en el aprendizaje de lenguas basado en un enfoque por tareas.

\section{Carga cognitiva}

Con frecuencia no será fácil distinguir lo que es cognitivamente difícil de conseguir para los aprendices de lo que es comunicativamente difícil. Sin embargo, podremos diseñar tareas en las que haya un aumento gradual de complejidad cognitiva, sin ampliar exageradamente la carga comunicativa. Por ejemplo, las tareas que requieren que los aprendices sigan una secuencia cronológica lógica, referidas a características personales o actividades individuales, exigirán mucho menos a nivel cognitivo que las tareas en las que no haya un desarrollo tan claro y en las que diversos actores y diversas acciones compliquen el escenario.

\section{Enfasis comunicativo}

En un manual para profesores de inglés hablado para extranjeros publicado recientemente, Yule y Brown (1983) describen una de las situacio-

* E.S.P.: English for Specific Purposes. 
nes más complejas en la que puede encontrarse un profesor no nativo. En dicha situación, el o la profesora debe conversar sobre un tema que es mucho más familiar a sus interlocutores, teniendo ellos una mayor competencia comunicativa y más conocimientos sobre el tema, sin que la organización de la tarea comunicativa esté claramente estructurada, y hallándose ésta más centrada en los motivos de la actuación que en la actuación en sí. Con un escenario tan poco adecuado, Yule y Brown (op. cit.), proponen la posible construcción de una secuencia de tareas comunicativas gradualmente más complejas, elevando la situación comunicativa inicial de tarea en tarea.

\section{Particularidades y posibilidad de generalizar}

Quizá nos gustaría poder asegurar que las tareas que siguen una estructura generalizada, como en el caso de tener contenidos asociados, o bien las que siguen algún esquema interpretativo ritualizado, serían más fáciles de manejar que aquéllas en las que el orden de asociación o las normas de interpretación no están claras y deben negociarse. Por ejemplo, seguramente será más difícil para cualquiera responder a preguntas originales sobre futuros acontecimientos hipotéticos que hacer referencia a experiencias cotidianas. Esa misma facilidad o dificultad puede rápidamente ser transportada a tareas de lectura o escritura, ofreciendo similares posibilidades de secuenciación. Sin duda querremos que los aprendices desarrollen la capacidad de manejar esas tareas rutinarias antes de que se enfrenten a actividades más creativas, a partir de los fines y procedimientos a los que ya se han adaptado.

\section{Complejidad de código y densidad interpretativa}

No hay una razón absoluta que explique que la complejidad del código requiera el correspondiente aumento de la densidad interpretativa del texto, pero es posible que queramos elaborar tareas, especialmente las centradas en la capacidad de comprensión lectora y auditiva, en las que sea posible una secuenciación, oponiendo la complejidad léxico-sintáctica a la "profundidad" de las preguntas interpretativas formuladas en la tarea. Por ejemplo, se pueden hacer preguntas más directas en textos complicados a nivel textual mientras que, en textos más sencillos, las preguntas pueden requerir que el aprendiz haga un análisis más explicativo e interpretativo. Dichas tareas sin duda ofrecerán un campo más amplio de preguntas sobre un mismo texto y serán adaptables a diversos niveles y estilos de aprendizaje.

\section{Continuidad del contenido}

Más arriba me he referido a la propuesta de Long (1984) sobre crear tareas en base a descripciones sociolingüísticas del objetivo de encuentro comunicativo. Ese tipo de procedimiento es ahora habitual en las tareas del E.S.P. Aunque el procedimiento de diseño de una tarea tal tiene ventajas aparentemente claras en lo que respecta a autenticidad de los objetivos, ya que derivan de la «vida real», frecuentemente hay un problema práctico dentro del aula: los participantes profesionales rechazan el tipo de encuen- 
tros simulados al percibir su falta de verosimilitud (Candlin, Bruton y Leather, 1976). Esta alineación psico-social a menudo va acompañada del hecho observado de que tales representaciones actúan como modelos que imposibilitan gran parte del compromiso activo y de la implicación de los aprendices (Breen, 1985). Sin embargo, parecería que para algunos aprendices, especialmente para los participantes en programas a corto plazo, ese diseño de tareas pudieran iniciarse fructíferamente a partir de esas tareas de contenido, permitiendo quizá una variedad en el tipo de trabajo que superase las críticas de Breen.

\section{Continuidad del proceso}

Con los materiales didácticos Challenges (Candlin y Edelhoff, 1982), se les ofrecen a los aprendices los medios para que creen la propia continuidad y secuenciación propia de las tareas posibles. Se estimula colectivamente a que los aprendices secuencien sus propias tareas tras examinar sus necesidades, que deben conocerse y experimentarse antes de realizar otra tarea en particular, y que para algunos será más compleja. En todo momento, durante la práctica, los aprendices reciben un feedback de la buena marcha del proceso de aprendizaje: el propio ( $₫$ Puedo ya iniciar esta nueva tarea?»), el de los compañeros, y el del profesor. Los aprendices se dan cuenta de que cada tarea necesita una preparación adecuada: formas de lenguaje, funciones y estrategias discursivas; formas de pensar y organizar las ideas; y conocimiento del contenido. Por medio de esa flexibilidad se posibilita que los alumnos organicen su orden de aprendizaje particular y que creen su propia continuidad.

\section{EVALUACION DE LAS TAREAS}

La idea original (Breen y Candlin, 1980) de que un currículum comunicativo llevado a cabo por tareas en el aula debiera poder ser autoevaluado ha recibido algunas críticas. Se considera que la evaluación es un proceso demasiado serio para ser dejado, como una guerra, en manos de profesores y aprendices. Había una necesidad de agentes externos (Rea, 1985). Tengo que rechazar esta necesidad disfrazada en forma de bien concedido, ya que una lectura detallada de las propuestas originales para un currículum comunicativo, junto con las sugerencias aquí presentadas, aclararán que una evaluación externa, que indudablemente puede tener valor de información corroborativa, es totalmente innecesaria en un programa en el que la actividad en el aula cuenta con un objetivo principal como lo constituye la evaluación formativa. Sin embargo, con el fin de proveer esta evaluación formativa, muy ilustrativa en el proceso de aprendizaje, será necesario que las tareas puedan valorarse de acuerdo a unos criterios previamente establecidos. ¿Cuáles serán esos criterios?

\section{Dificultades}

¿Hasta qué punto una tarea determinada muestra la variación entre las destrezas y los conocimientos de los aprendices? ¿De qué forma puede ser diagnóstica y explicativa? Además de la corrección (véase p. 40-41), ¿̇pro- 
porciona la tarea un feedback de tal forma que puedan mostrarse las posibles rutas de actuación futura, adecuadas para cada aprendiz en particular, o para toda la clase? ¿Hasta qué punto ofrece la tarea posibilidades para futuras actividades?

\section{Posibilidad de implementación}

¿Cuáles son las exigencias de la tarea respecto a los recursos necesarios? ¿Qué complejidad de organización en el aula y de dirección, profesor-aprendiz, se presupone? ¿Qué requisitos logísticos impone la tarea? ¿Podemos evaluar la implementación de las tareas a dos niveles: a nivel de contenido de la tarea y a nivel de acción de la tarea? Podemos idear tareas en las que las diversas rectificaciones del proceso de trabajo de la tarea influyan sobre la naturaleza y extensión del contenido procesado. En otras palabras, las tareas pueden evaluarse de acuerdo a su capacidad de adaptarse $y$ conformarse a la contribución del aprendiz.

\section{Posibilidad combinatoria}

Las tareas se diferenciarán entre sí según las posibilidades que ofrezcan para ser combinadas con otras tareas. Algunas constituirán verdaderas tareas procesuales: serán neutras en lo que respecta al contenido y podrán introducirse y adaptarse dentro del programa en puntos muy diversos según requieran los aprendices. Ese tipo de tareas libres e independientes se basan en las sugerencias de diseño de materiales para un currículum comunicativo presentadas ahora hace algún tiempo por Breen, Candlin y Waters (1979), denominadas aquí tareas procesuales. Otras dependerán en mayor parte de alguna secuencia de tareas, actuando como mecanismos posibilitadores de la ejecución de fines más complejos. Con el tiempo, naturalmente, y con la ayuda de los informes retrospectivos referidos al principio de este trabajo, algunas de las tareas tendrán un lugar determinado dentro de la secuenciación del aprendizaje de cada aprendiz, asociadas posiblemente, tras una práctica eficaz, a problemas de aprendizaje concretos, o a dificultades especiales dentro del contenido. La posibilidad de combinación no es, pues, algo dado: emerge a través de la asegurada evaluación formativa de los acuerdos del programa, como medio de evaluar las tareas.

\section{EPILOGO}

Soy consciente de que todo lo que expongo más arriba plantea muchas cuestiones en lugar de ofrecer conclusiones. Quedan muchos puntos por abordar. No he sacado a colación la problemática de la asignación de papeles de los participantes en determinadas tareas, ni he examinado en minucioso detalle la contribución que las tareas pueden aportar en la definición del material de trabajo, del programa y, también, del currículum. Ello se debe a que, en la enseñanza de la comunicación, las conveniencias se negocian de forma paralela al proceso de aprendizaje, y las tareas tienen ahí un papel codificante. Sin embargo, lo que espero haber conseguido es ofrecer, quizá a propósito, las siguientes recompensas de un aprendizaje de lenguas basado en un enfoque por tareas: 
- Una reestructuración de la relación entre el currículum y los programas.

- Una estimación distinta de la relación entre datos, recursos y procesos en el contenido de la tarea y la acción en la tarea.

- Una revaloración de la relación entre un tipo de evaluación basada en fines y otra referida a normas.

- Una revisión de la relación de los papeles del «profesor» y del «aprendiz" en la participación mutua dentro de la tarea.

- Un nuevo énfasis del potencial del aula como lugar de experimentación de lenguaje y del aprendizaje del lenguaje.

- Una renovación de una perspectiva crítica sobre la comprensión de la adquisición de una segunda lengua y el papel del aprendizaje de lenguas en la identidad social del aprendiz.

\section{Referencias}

BREEN, M. P. (1983). «How would we recognise a communicative classroom». Dunsford House Papers. The British Council.

BREEN, M. P. (1984). "Processes in Syllabus design", en C. Brumfit (ed.), General English Syllabus Design, ELT Documents 118, Londres: Pergamon Press, and British Council.

BREEN, M. P. (1985a). "Authenticity in the language classroom», Applied Linguistic, 6/1, 60-70.

BREEN, M. P. y CANDLIN, C. N. (1980). "The essentials of a communicative curriculum in language teaching», Applied Linguistic, 1/2, 89-112.

BREEN, M., CANDLIN, C. N. y WATERS, A. (1979). «Communicative materials design: some basic principles», RELC Journal, 10 December 1979.

BRUNER, J. (1974). «From communication to language. A psychological perspective», Cognition, $1974 / 5,3$.

CANALE, M. (1983). «From communicative competence to communicative language teaching pedagogy», en J. Richards y J. Schmidt (eds.), Language and Communication, Londres: Longman, 1983.

CANALE, M. y SWAIN, M. (1980). «Theoretical bases of communicative approaches to second language teaching and testing», Applied Linguistics, 1/1.

CANDLIN, C. N. (1984). «Syllabus design as a critical process", en C. Brumfit (1984), op. cit.

CANDLIN, C. N. (1985). "Explaining communicative competence: the limits of testability", en Report 21 of the IInd International TOEFL Conference, ETS, Princeton, New Jersey.

CANDlin, C. N. y EDELHOFF, C. (1982). Challenges: Teachers' Guide, Londres: Longman.

DAM, L. (1983). Beginning English - an experiment, Danmarks Laererhejskole/Greve Kommune, Copenhagen, Denmark (mimeo).

DAM, L. (1984). Intermediate English, Danmarks Laererhøjskole/Greve Kommune, Copenhagen, Denmark (mimeo).

DAM, L. (1985). «Strategies for the correction of errors in different teaching situations and their implications for initial and in-service teacher training", Greve Kommune, Copenhagen, Denmark (mimeo).

FAERCH, C. y KASPER, G. (1983) (eds.). Strategies of Interlanguage Communication, Londres: Longman.

GREVE (1981). Report of a workshop on communicative curriculum design (L. Dam, M. P. Breen y C. N. Candlin) Greve Kommune, Copenhagen, Denmark (mimeo).

Johnson, K. y PORTER, D. (1983) (eds.). Perspectives in Communicative Language Teaching, Londres: Academic Press.

LEECH, G. N. y CANDLIN, C. N. (1986) (eds.). Computers in English Language Teaching and Research, Londres: Longman.

LONG, M. (1984). *The design of classroom second language acquisition: towards task-based language teaching», en K. Hyltenstam y M. Pienemann (eds.), Instructural and Social Implications of Second Language Acquisition research, Multilingual Matters, Cleveden, Avon.

PrabHu, N. (1983). «Procedural Syllabuses». Paper given at the 18th RELC Seminar, Singapore, 1983 (mimeo).

RATHS, J. D. (1971). «Teaching without specific objectives», Educational Leadership, April, 714-720.

REA, P. M. (1985). «Language testing and the communicative language curriculum», en Y. Lee, 
A. Fok, R. Lord y G. Low (eds.), New Directions in Language Testing, Oxford, Pergamon Press.

RuBIN, J. (1981). *Study of cognitive processes in second language learning*, Applied Linguistic, 11/2, 1981.

RUTHERFORD, W. (1986). Second Language Grammar: Learning y Teaching, Londres: Longman.

SHARWOOD-SMITH, M. (1981). «Consciousness-raising and the second language learner*, Applied Linguistic, 2.

STENHOUSE, L. (1975). An introduction to Curriculum Research and Development, Londres: Heinemann.

WATERS, A. y Hutchinson, T. (1983). «ESP at the Crossroads», en J. Swales (1985), Episodes in ESP, Londres: Pergamon.

WENDEN, A. (1985). "Incorporating learner training into the classroom», en A. Wenden y J. Rubin (eds.), Learner Strategies, Pergamos Press (forthcoming, 1986).

WIDdowson, H. (1983). Learning Purpose and Language Use, Oxford: Oxford University Press.

YULE, G. y BRown, G. (1983). Teaching the Spoken Language, Cambridge University Press.

Hacia la enseñanza de lenguas basada en tareas. C. $N$. Candlin. CLEE, 1990, 7-8, pp. 33-53.

Datos sobre el autor: Christopher Candlin es un representativo autor del denominado enfoque de enseñanza por tareas. Trabaja en el Departamento de Lingüística y Enseñanza de Inglés Moderno en la Universidad de Lancaster.

Dirección: University of Lancaster, Department of Linguistic and Modern English Language, Lancaster, LA14Y'T, Gran Bretaña.

Título original: Towards task-based language learning, Lancaster Practical Papers in English Languaje Education, 1987, 7, 5-22. Reproducido con autorización. Traducción de Margarita Hernández.

(C) De todos los artículos deberá solicitarse por escrito autorización de CL\&E y de los autores para el uso en forma de facsímil, fotocopia o cualquier otro medio de reproducción impresa. CL\&E se reserva el derecho de interponer las acciones legales necesarias en aquellos casos en que se contravenga la ley de derechos de autor. 